\title{
KNOTTED SYMMETRIC GRAPHS
}

\author{
CHARLES LIVINGSTON
}

(Communicated by Ronald Stern)

\begin{abstract}
For a knotted graph in $S^{3}$ we define the vertex constant group, a quotient of the fundamental group of the complement. For planar graphs the group is cyclic. For graphs with periodic symmetry the group is related to the fundamental group of the branched cover of $S^{3}$ branched over knots contained in the quotient of the graph under the symmetry. These tools are used to demonstrate that a large family of knotted graphs are not planar.
\end{abstract}

This note presents a new tool for distinguishing knotted graphs in $S^{3}$. To each such graph $\Gamma$ we associate a group, called the vertex constant group; for connected planar graphs the vertex constant group is finite cyclic. Our first application is to offer a new proof that all elements of a particular family of knotted graphs, denoted $\vartheta_{n}$, are nontrivial. This family of graphs was introduced by Suzuki [Su], expanding on an example of Kinoshita [Ki]; it is of particular interest as a test case for new methods since each element is Brunnian-that is, every subgraph is planar. Suzuki proved that the $\vartheta_{n}$ are nontrivial using an algebraic argument in which he showed that the fundamental group of the complement is not free; Scharlemann [S] has described a more geometric approach, reducing the problem to a calculation in the braid group.

Our second application is to demonstrate that the $\vartheta_{n}$ represents a special case of a large class of knotted graphs, all of which will be proved nontrivial without any explicit calculation. Neither of the approaches of [S, Su] nor apparently any other known techniques apply to the entire class. (See [K1, K2, T, Y] for recent progress in the study of knotted graphs.) Figure 1 illustrates the graph $\vartheta_{5}$. One vertex has been placed at the point at infinity to highlight the periodic symmetry of the graph. Similarly, $\vartheta_{n}$ has two vertices joined by $n$ edges. Note that there is a period $n$ transformation, $T$, of $S^{3}$ leaving $\vartheta_{n}$ invariant. This action determines a branched cover of $S^{3}$, the image of $\vartheta_{n}$, is an arc, $K$. Figure 2 illustrates $K$ along with the branch set, described as the union of two arcs, $B_{1}$ and $B_{2}$. The union of $B_{2}$ and $K$ is a trefoil knot. We will see that if the edges of $\vartheta_{n}$ are replaced with any other periodic family of edges, the graph continues to be nontrivial, as long as the union of $B_{2}$ and $K$ forms a nontrivial knot in $S^{3}$.

Received by the editors June 14, 1993.

1991 Mathematics Subject Classification. Primary 57M25; Secondary 57M60.

Key words and phrases. Knotted graphs. 
964

CHARLES LIVINGSTON

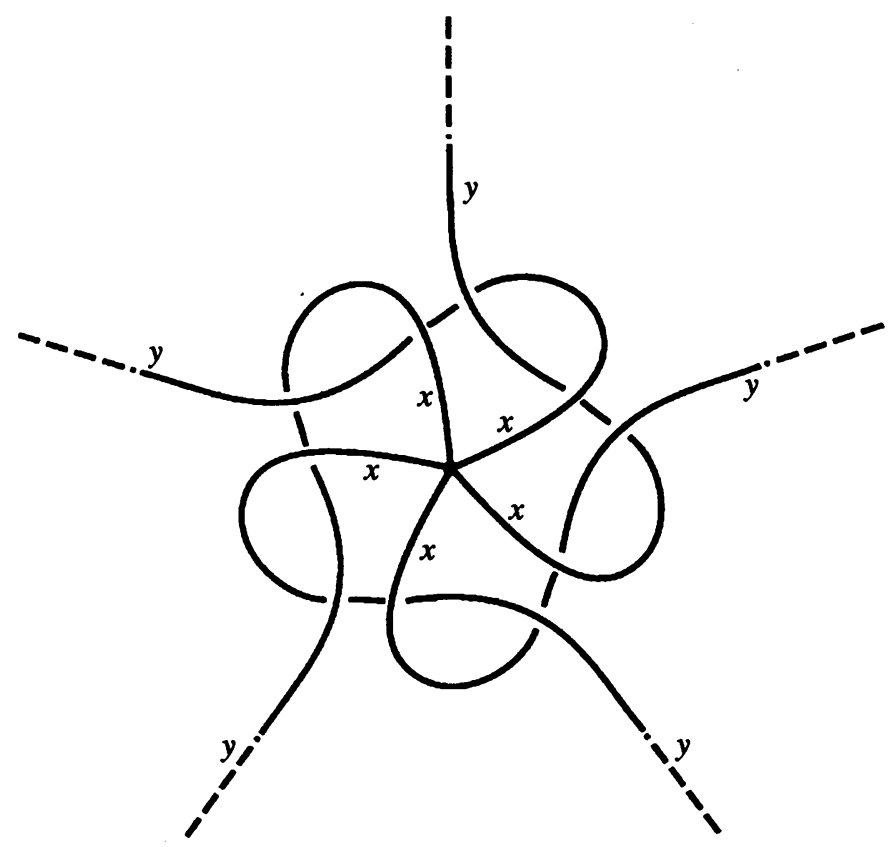

Figure 1

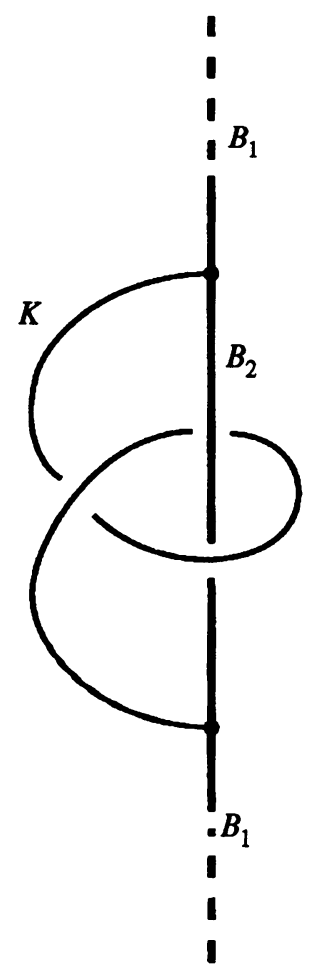

Figure 2 


\section{THE VERTEX CONSTANT GROUP}

Given a regular projection of a knotted graph $\Gamma$ there is an associated Wirtinger presentation of the fundamental group of the complement, $\pi$, defined as for knots. The vertex constant group, $\pi^{*}$, is the quotient of $\pi$ defined by setting all meridians at each vertex equal. For this to be well defined an orientation of each meridian near each vertex must be chosen. This is done by (locally) orienting each edge of the graph near the vertex to point toward that vertex. As one example, Figure 1 includes Wirtinger generators of the graph $\vartheta_{5}$. The generators at each vertex have been identified and labelled $x$ and $y$. A quick calculation shows the vertex constant group has presentation

$$
G_{n}=\left\langle x, y \mid x y^{-1} x^{-1}=y x y^{-1}, x^{n}=1\right\rangle \text {. }
$$

(For details on the notation of the figure, and of the method of calculation, see $[\mathrm{C}]$.)

Proving that the isomorphism class of $\pi^{*}$ depends only on the isotopy class of $\Gamma$ is a standard exercise in knot theory. Note however that the vertex constant group is not an invariant of the complement of the graph, or even of the topological pair $\left(S^{3}, \Gamma\right)$; adding a vertex to an edge adds the relation that the square of the meridian to that edge is trivial.

For a connected planar graph the vertex constant group is easily seen to be cyclic; more specifically, if $\vartheta_{n}$ were planar, then $G_{n}$ would be isomorphic to $Z_{n}$. Here is a quick way to prove this is not the case. Observe that $G_{n}$ is isomorphic to the group of the trefoil knot with the added relation that the $n$th power of the meridian is trivial. (In the usual presentation of the trefoil group $y$ is replaced with $y^{-1}$ in the above presentation.) There is a natural homomorphism onto $Z_{n}$; the kernel is the fundamental group of the $n$-fold cover of the trefoil complement with the added relation that the lift of the meridian to that cover is trivial. That is, the kernel of the map onto $Z_{n}$ is the fundamental group of the $n$-fold branched cover of the trefoil. This group is known to be nontrivial. (For the trefoil this can be shown explicitly; it also follows from the Smith Conjecture [B].)

\section{PERIODIC GRAPHS}

We now want to see that replacing the edges of $\vartheta_{n}$ with a different periodic set of edges will generally leave the graph nonplanar. We begin by offering a slightly different proof that $\vartheta_{n}$ is nontrivial, one that exploits the periodicity of the graph, and then note that this alternative proof immediately extends to the more general setting.

There is a period $n$ transformation, $T$, on the complement of $\vartheta_{n}$ with fixed set a pair of arcs, $B_{1}^{*}$ and $B_{2}^{*}$. The quotient of this action is the complement of the arc $K$ in $S^{3}$, illustrated in Figure 2. The images of the branch curves in the quotient are also shown in Figure 2, labelled $B_{1}$ and $B_{2}$. Note that $K \cup B_{2}$ is the trefoil knot. As will soon be seen, the appearance of the trefoil knot and the role of the trefoil in the previous argument are not coincidental.

Pick a basepoint for $S^{3}-\vartheta_{n}$ on the branch set, $B_{1}^{*}$. Then $T$ defines an action on $\pi$ denoted $T_{*}$. Define a quotient of $\pi, \pi^{\#}$, by adding the relations $x=T_{*}(x)$ for all $x$ in $\pi$. Clearly $\pi^{\#}$ is a quotient of $\pi^{*}$, so showing that $\pi^{\#}$ is noncyclic will imply that $\pi^{*}$ is noncyclic. A covering space argument 
presented in the next section shows that $\pi^{\#}$ is isomorphic to the group of the complement of the knot $K \cup B_{2}$ with the added relation that the $n$th power of the meridian to $B_{2}$ is trivial. But, as in the argument in the previous section, this group has an index $n$ subgroup isomorphic to the fundamental group of the $n$-fold branch cover of $S^{3}$ branched over $K \cup B_{2}$. Hence, by the Smith Conjecture, $\vartheta_{n}$ is nontrivial.

This proof just given reveals that the essential property of the $\vartheta_{n}$ is that $K \cup B_{2}$ is knotted. Hence, if the edges of $\vartheta_{n}$ are replaced with any other periodic family, the graph remains nontrivial, as long as $K \cup B_{2}$ is nontrivial.

\section{COVERING SPACES}

We conclude with the covering space argument used in the previous section to analyze $\pi^{\#}$.

Suppose that $p:\left(M, B_{1}^{*}, \ldots, B_{k}^{*}\right) \rightarrow\left(N, B_{1}, \ldots, B_{k}\right)$ is an $n$-fold cyclic branched covering of 3-manifolds, branched over arcs $B_{1}, \ldots, B_{n}$, each of branch index $n$. Let $T$ denote the generator of deck transformations, and let $\pi^{\#}$ denote the quotient of $\pi_{1}\left(M, b_{1}^{*}\right)$ under the relations $x=T_{\#}(x)$ for all $x$, where $b_{1}^{*}$ is a basepoint on $B_{1}^{*}$.

Theorem. The group $\pi^{\#}$ is isomorphic to the quotient of $\pi_{1}\left(N-\left(B_{2} \cup \cdots \cup B_{n}\right), b_{1}\right)$ under the relations $m_{i}^{n}=1, i>1$, where the $m_{i}$ are the meridians to the $B_{i}$. Proof. First consider the case that $k=1$. The projection map from $\pi_{1}\left(M, b_{1}^{*}\right)$ to $\pi_{1}\left(N, b_{1}\right)$ is easily seen to determine a well-defined map of the quotient $\pi^{\#}$ to $\pi_{1}\left(N, b_{1}\right)$. Next observe that a lifting argument implies that this induced map is surjective. To prove injectivity, represent an element in the kernel by a path $\gamma^{*}$. By transversality it can be assumed that $\gamma^{*}$ is disjoint from $B_{1}^{*}$ except at its endpoints. Let $\gamma$ be the image of $\gamma^{*}$ in $N$. Again using transversality it can be assumed that $\gamma$ bounds a disk in $N$ that meets $B_{1}$ transversely, and after an isotopy, all points of intersection of the disk with $B_{1}$ are at $b_{1}$. Hence, $\gamma$ is homotopic, via a homotopy missing $B_{1}$ to a product of paths, based at $b_{1}$, each of the form $\omega m_{1} \omega^{-1}$ where $\omega$ is a closed path in $N$ meeting $B_{1}$ only at its endpoints. This homotopy lifts to the branched cover. The lift of each individual path is of the form $x T_{\#}\left(x^{-1}\right)$. (The element $x$ is represented by a lift of $\omega$.) Hence, $\gamma^{*}$ represents the trivial element in the quotient group $\pi^{\#}$.

In the case that $k>1$, consider the manifolds constructed by removing all the components of the branch set of $M$, other than $B_{1}$, and the corresponding branch curves from the quotient. The argument of the previous paragraph applies for these subspaces. The full spaces are reconstructed by adding in the branch curves. Since the meridians of the branch curves in the cover are identified, via the isomorphism, with the $n$th powers of meridians of the branch curves in the quotient, an isomorphism is maintained if these powers of the meridians in the quotient are set equal to the identity.

\section{REFERENCES}

[B] H. Bass and J. Morgan, The Smith Conjecture, Academic Press, Orlando, FL, 1984.

[C] R. Crowell and R. Fox, Introduction to knot theory, Graduate Texts in Math., vol. 57, Springer-Verlag, New York, 1977.

[K1] L. Kauffman, Invariants of graphs in 3-space, Trans. Amer. Math. Soc. 311 (1988), 697-710. 
[K2] L. Kauffman, J. Simon, K. Wolcott, and P. Zhao, Invariants of theta-curves and other graphs in 3-space, Topology Appl. 49 (1993), 193-216.

[Ki] S. Kinoshita, On elementary ideals of polyhedra in the 3-sphere, Pacific J. Math. 42 (1972), 89-98.

[S] M. Scharlemann, Some pictorial remarks on Suzuki's Brunnian graph, Topology '90-Proc. of Ohio State Topology Year (B. Apanasov, W. D. Neumann, A. W. Reid, and L. Siebenmann, eds.), Walter de Gruyter, Berlin, 1992, pp. 351-354.

[Su] S. Suzuki, Almost unknotted $\vartheta_{n}$-curves in 3-sphere, Kobe J. Math. 1 (1984), 19-22.

[T] A. Thompson, Polynomial invariants of graphs in 3-manifolds, Topology 31 (1992), 657-665.

[Y] S. Yamada, A topological invariant of spatial regular graphs (A. Kawauchi, ed.), Knots 90, Walter de Gruyter, New York, 1992.

Department of Mathematics, INDiana UNiversity, BLoOMington, INDiana 47405

E-mail address: livingst@math.indiana. edu 